\title{
Bintrafusp Alfa, a Bifunctional Fusion Protein Targeting TGF- $\beta$ and PD-L1, in Patients with Esophageal Adenocarcinoma: Results from a Phase 1 Cohort
}

\author{
Benjamin Tan ${ }^{1}$ - Adnan Khattak ${ }^{2}$ - Enriqueta Felip ${ }^{3} \cdot$ Karen Kelly $^{4} \cdot$ Patricia Rich $^{5,6}$. Ding Wang ${ }^{7} \cdot$ Christoph Helwig $^{8}$. \\ Isabelle Dussault ${ }^{9,10} \cdot$ Laureen S. Ojalvo $^{9,10} \cdot$ Nicolas Isambert $^{11}$
}

Accepted: 19 March 2021 / Published online: 19 May 2021

(c) The Author(s) 2021

\begin{abstract} to a human IgG1 mAb blocking PD-L1. treated, advanced esophageal adenocarcinoma.

Clinical Trials Registration NCT02517398.

\section{Background}

In 2018, esophageal cancer caused approximately 500,000 deaths worldwide [1]. The prognosis of patients with esophageal cancer is poor, with age-standardized 5-year survival rates ranging from 10 to $30 \%$ after correction for background mortality [2]. The majority of patients have advanced disease at diagnosis, with 5-year survival rates below 10\% [3].

Esophageal cancer comprises two subtypes: adenocarcinoma (AC) and squamous cell carcinoma (SCC). Although SCC remains the most prevalent histological type of esophageal cancer, incidence rates are decreasing worldwide [1]. In contrast, the incidence of AC is rapidly increasing in Western countries such as Australia, England, France, and the
\end{abstract}

Background Esophageal adenocarcinoma patients have limited treatment options. TGF- $\beta$ can be upregulated in esophageal adenocarcinoma, and blocking this pathway may enhance clinical response to PD-(L)1 inhibitors. Bintrafusp alfa is a firstin-class bifunctional fusion protein composed of the extracellular domain of the TGF- $\beta$ RII receptor (a TGF- $\beta$ "trap") fused

Objective The objective of this study was to investigate the efficacy and safety of bintrafusp alfa in patients with advanced, post-platinum esophageal adenocarcinoma, unselected for PD-L1 expression.

Patients and Methods In this phase 1 study, patients with post-platinum, PD-L1-unselected esophageal adenocarcinoma received bintrafusp alfa $1200 \mathrm{mg}$ every 2 weeks until disease progression, unacceptable toxicity, or withdrawal. The primary endpoint was confirmed best overall response per RECIST 1.1 by independent review committee (IRC).

Results By the database cutoff of 24 August 2018, 30 patients (80.0\% had two or more prior anticancer regimens) received bintrafusp alfa for a median of 6.1 weeks. The confirmed objective response rate (ORR) per IRC was 20.0\% (95\% CI 7.738.6); responses lasted 1.3-8.3 months. Most responses (83.3\%) occurred in tumors with an immune-excluded phenotype. Investigator-assessed confirmed ORR was $13.3 \%$ (95\% CI 3.8-30.7). Nineteen patients (63.3\%) had treatment-related adverse events: seven patients (23.3\%) had grade 3 events; no grade 4 events or treatment-related deaths occurred.

Conclusions Bintrafusp alfa showed signs of clinical efficacy with a manageable safety profile in patients with heavily pre-

Nicolas Isambert

nicolas.isambert@chu-poitiers.fr

Extended author information available on the last page of the article
USA [1]. This observed increase is most likely due to the escalating prevalence of risk factors for esophageal AC, such as obesity and chronic gastroesophageal reflux disease $[1,4]$.

Genetic profiling has identified tumor-specific alterations in esophageal AC affecting ERBB2, VEGFA, GATA4, and GATA6, as well as genes involved in DNA hypermethylation, but other factors in the tumor microenvironment (TME) are also known to impact cancer [5-7]. Transforming growth factor $\beta$ (TGF- $\beta$ ) is one such factor in the TME. TGF- $\beta$ is a cytokine with highly diverse functions that can act in either a tumor-suppressive or a tumor-promoting role, depending on the tumor type and stage, and genetic and/or epigenetic changes within the TGF- $\beta$ pathway $[8,9]$. Signaling through the TGF- $\beta$ pathway can enable cancer progression and immune evasion in the TME through regulatory effects on immune cells, and may also influence angiogenesis and epithelial-to-mesenchymal transition (EMT) $[9,10]$. Preclinical studies have shown that TGF- $\beta$ can induce EMT in 


\section{Key Points}

Bintrafusp alfa is a first-in-class bifunctional fusion protein that was designed for co-localized, simultaneous inhibition of two nonredundant immunosuppressive pathways, TGF- $\beta$ and PD-L1, within the tumor microenvironment.

Treatment with bintrafusp alfa had a manageable safety profile and demonstrated clinical activity in patients with advanced, post-platinum esophageal adenocarcinoma from a phase 1 expansion cohort.

These results, along with those reported in the accompanying article from an expansion cohort of Asian patients with pretreated, PD-L1-unselected esophageal squamous cell carcinoma in a separate phase 1 study, support further clinical investigation of bintrafusp alfa in esophageal cancer.

esophageal AC cell lines and that expression of TGF- $\beta$ correlated with the expression of EMT-related genes [11, 12]. Furthermore, elevated expression of TGF- $\beta$ in esophageal $\mathrm{AC}$ has been associated with advanced stages of disease, and correlates with poor overall survival (OS) [13].

Antibodies that target immune checkpoints have emerged in recent years as a novel therapeutic approach for the treatment of a wide range of cancers. The efficacy of antiPD-1 agents has been investigated in advanced, pretreated esophageal cancer; however, these studies often had low enrollment of patients with esophageal $\mathrm{AC}[14,15]$. In the KEYNOTE-180 and KEYNOTE-181 clinical trials, pembrolizumab showed an objective response rate (ORR) of 5\% in patients with PD-L1-unselected (all-comer), 3\% in those with PD-L1-negative (combined positive score [CPS] < 10), and $18 \%$ in those with PD-L1-positive (CPS $\geq 10$ ) esophageal AC $[14,15]$.

Preclinical studies have suggested that the efficacy of antiPD-(L) 1 agents may improve with the addition of TGF- $\beta$ blockade [16-19]. Bintrafusp alfa is a first-in-class bifunctional fusion protein composed of the extracellular domain of the human TGF- $\beta$ receptor II (TGF- $\beta$ RII or TGF- $\beta$ “trap") fused via a flexible linker to $C$-terminus of each heavy chain of IgG1 antibody blocking PD-L1 (anti-PD-L1) [20]. Bintrafusp alfa is designed for co-localized, simultaneous inhibition of two nonredundant immunosuppressive pathways (TGF- $\beta$ and PD-L1) within the TME [20]. This may provide an enhanced treatment effect, potentially improving clinical benefit compared with anti-PD-(L)1 monotherapies [20-22].

Clinical data from two phase 1 studies of bintrafusp alfa (NCT02517398 and NCT02699515) have demonstrated a manageable safety profile and early signs of clinical efficacy in patients with heavily pretreated advanced solid tumors [23-25]. Here we report results from an expansion cohort of the phase 1 study, which investigated the efficacy and safety of bintrafusp alfa in patients with advanced, post-platinum esophageal AC, unselected for PD-L1 expression.

\section{Patients and Methods}

\subsection{Study Design and Participants}

This phase 1, open-label trial investigated the safety and efficacy of bintrafusp alfa in patients with heavily pretreated solid tumors and included multiple expansion cohorts in specific tumor types (NCT02517398). Patients were screened across the Asia-Pacific region, Europe, Canada, and the USA.

Eligible patients had histologically confirmed recurrent or metastatic, unresectable (stage III/IV) esophageal AC and measurable disease by Response Evaluation Criteria in Solid Tumors version 1.1 (RECIST 1.1). Patients were at least 18 years old and must have received at least one prior platinumbased regimen. Patients with HER2-positive tumors must have received prior trastuzumab. Other inclusion criteria included Eastern Cooperative Oncology Group (ECOG) performance status of 0 or 1 , life expectancy of $\geq 12$ weeks, and adequate renal, hepatic, and hematological function. Prior to enrollment, tumor samples (archival material or fresh biopsies obtained within 28 days) were collected from each patient; however, no selection was made on the basis of PD-L1 expression or other biomarkers. Prior therapy with immune checkpoint inhibitors was not permitted.

\subsection{Treatment and Assessments}

Patients received bintrafusp alfa at the recommended phase 2 dose of $1200 \mathrm{mg}$ intravenously over $1 \mathrm{~h}$ once every 2 weeks until confirmed progressive disease, unacceptable toxicity, or trial withdrawal [26]. A flat dose of $1200 \mathrm{mg}$ every 2 weeks was selected as the recommended phase 2 dose of bintrafusp alfa based on safety/tolerability, pharmacokinetic, and pharmacodynamic data, as well as preliminary population pharmacokinetics and exposure-response modeling of phase 1 data [26, 27]. The planned duration of treatment for patients who achieved a best overall response of complete response, partial response, or stable disease was 12 months. If the investigator believed that a patient may benefit, additional treatment beyond 12 months could be possible. Dose reductions were not permitted. Tumor responses were monitored by imaging every 6 weeks during the first year and every 12 weeks thereafter. Scans were reviewed by an independent review committee to assess disease response. Efficacy and safety were analyzed in all patients who received at 
least one dose of bintrafusp alfa. Adverse events (AEs) were monitored throughout treatment and were assessed according to National Cancer Institute Common Terminology Criteria for Adverse Events (NCI-CTCAE) version 4.03. An additional safety follow-up visit was planned 28 days after the last study dose or before the start of a new treatment (whichever occurred first). Treatment-related AEs (TRAEs) continued to be evaluated 10 weeks post-treatment. Any AE that was believed to be a potential immune-related or potential TGF- $\beta$-related event was considered an AE of special interest. Immune-related AEs (irAEs) were identified using a preselected list of terms from the Medical Dictionary for Regulatory Activities version 21.0.

\subsection{Endpoints}

The primary endpoint was confirmed best overall response according to RECIST 1.1 as adjudicated by the independent review committee and evaluated by the confirmed ORR; key secondary endpoints included the safety of bintrafusp alfa and best overall response per investigator assessment. Other efficacy measures included duration of response, disease control rate (DCR), progression-free survival (PFS), and OS. The evaluation of potential predictive biomarkers was an exploratory endpoint.

\subsection{Exploratory Endpoints}

PD-L1 expression was detected by immunohistochemistry staining of formalin-fixed, paraffin-embedded tumor tissue using an anti-PD-L1 antibody clone 73-10 (Dako PD-L1 IHC 73-10 pharmDx; Dako, Carpinteria, CA, USA). PD-L1 expression was measured on tumor cells and on cells of the TME. Data herein are reported based on the percentage of tumor cells expressing PD-L1. A threshold of $1 \%$ was used to characterize tumors as either PD-L1 positive $(\geq 1 \%)$ or PD-L1 negative $(<1 \%)$.

RNA sequencing (RNAseq) was also performed to assess the correlation between specific tumor characteristics and response to bintrafusp alfa. RNAseq used formalin-fixed, paraffin-embedded archival tumor samples and was performed by Asuragen (Austin, TX, USA) using standard protocols based on ribosomal depletion. Resulting sequencing reads were aligned against the Ensembl 75 human genome (GRCh37 February 2014) with Bowtie2 version 2.2.3 (Johns Hopkins University, Baltimore, MD, USA) [28]. Gene expression was determined using RSEM version 1.2.31 and Ensembl gene annotations, and hypothesis testing was performed by comparing RSEM-computed expected counts [29]. Transcript-per-million values were upper-quartile normalized and log transformed for further analysis. To test whether $T G F B 1, T G F B 2$, and $T G F B 3$ gene expression was higher in this cohort than in other expansion cohorts across phase 1 trials of bintrafusp alfa (NCT02517398 and NCT02699515), we applied limma + voom, modeling expression as a function of indication [30]. The analysis included all patient samples that passed quality control $(N$ $=537$, including 27 esophageal AC samples) and tested all genes with $\geq 10$ reads in $\geq 20$ samples. We report $p$ values for differential expression after adjustment for testing of all genes.

Tumor mutation count was measured by an RNAseqbased variant calling that used tumor RNAseq data combined with germline, normal whole-exome sequencing to produce a set of tumor-specific mutations. Tumor samples were sequenced at $2 \times 50$ to a target of $10^{8}$ read pairs with an Illumina HiSeq System (Illumina, San Diego, CA, USA). Whole-exome sequencing was performed by Expression Analysis (Research Triangle Park, NC, USA) using matched peripheral blood samples and an Agilent SureSelect Human All Exon V5 kit (Agilent Technologies, Santa Clara, CA, USA). Sequencing was done on an Illumina HiSeq System with a target of $100 \times$ coverage. Sequencing reads were mapped to hg19 and the Ensembl gene annotations (ensGene; University of California, Santa Cruz, CA, USA) using RNA-STAR version 2.5.0b; whole-exome reads were mapped to hg19 using BWA-MEM version 0.7.12 [31, 32]. Mutation calling was performed on paired BAM files using VarDictJava version 1.4.2 [33]. Results were annotated using Ensembl Variant Effect Predictor version 85 to determine the location and type of mutation [34]. Tumor mutation count was defined for each patient as the total count of all missense mutations discovered for that sample.

Immune phenotype was determined from tumor samples using available immunohistochemistry data (PD-L1 stain and negative control) and hematoxylin and eosin stain. A pathologist who was masked to the response data scored the scanned images and determined the corresponding immune phenotype using an exploratory classification system [35-38]. Inflamed samples had immune cells in direct physical contact with tumor cells. Immune-excluded samples had $\geq 1 \%$ of the tumor stroma area populated by lymphocytes where they may be near tumor cells without infiltration and infrequent physical contact between lymphocytes and tumor cells. Immune-desert samples had $<1 \%$ of the tumor stroma area populated by lymphocytes, with no dense immune cell infiltrates and no contact between immune and tumor cells.

\subsection{Statistical Analysis}

Thirty patients were planned for this expansion cohort to give the study approximately $87 \%$ power to rule out $\leq 15 \%$ ORR (null hypothesis) when the true ORR is $35 \%$ with 1 -sided $\alpha=0.1$. The analysis presented here was performed based on a data cutoff approximately 1.5 years after the last patient enrolled in the study. The confirmed ORR was 
defined as the proportion of patients achieving a confirmed complete or partial response. The uncertainty of the estimates was assessed by a two-sided 95\% Clopper-Pearson CI. DCR was defined as the proportion of patients with a best overall response of complete response, partial response, stable disease, or non-complete response/non-progressive disease. PFS and OS time were analyzed using the KaplanMeier method.

\section{Results}

\subsection{Patient Demographics and Characteristics}

Between 12 September 2016, and 31 January 2017, 47 patients with advanced, post-platinum esophageal AC who had disease progression after at least one prior line of treatment were screened; 30 patients from 21 centers met the eligibility criteria and were treated with bintrafusp alfa. Of these patients, 14 (46.7\%) were from the USA, ten (33.3\%) were from Europe, five (16.7\%) were from Australia, and one $(3.3 \%)$ was from Taiwan. The median age was 61 (range 30-80) years, and most patients had an ECOG performance status of $1(n=25$ [83.3\%]). This was a heavily pretreated population; $80.0 \%$ of patients had received at least two prior anticancer therapy regimens, and $36.7 \%$ of patients had received at least two prior lines of therapy for locally advanced or metastatic disease. Most tumors ( $n$ $=20[66.7 \%]$ ) were negative for PD-L1 expression, with nine $(30.0 \%)$ being positive and one $(3.3 \%)$ that was not evaluable. Detailed baseline characteristics are described in Table 1.

As of 24 August 2018, 30 patients received bintrafusp alfa for a median duration of 6.1 (range 2.0-55.7) weeks. The Kaplan-Meier estimate of median duration of follow-up since first dose was 86.1 (range 1.3-91.4) weeks. All patients had discontinued treatment at the time of data cutoff. Reasons for discontinuation were disease progression $(n=19$ [63.3\%]), AEs $(n=8[26.7 \%])$, death $(n=2[6.7 \%])$, and other reasons ( $n=1$ [3.3\%]; patient completed 12 months' treatment). Following treatment with bintrafusp alfa, eight patients $(26.7 \%)$ received at least one type of subsequent anticancer treatment, of whom six patients received cytotoxic therapy.

\subsection{Efficacy}

Responses occurred in six patients per independent review committee assessment, all of which were partial responses (confirmed ORR, 20.0\% [95\% CI 7.7-38.6]) (Table 2, Fig. 1a). The median duration of response was 4.3 (range 1.3-8.3) months (Table 2, Fig. 1b). An additional four patients had stable disease (DCR, 33.3\% [95\% CI
Table 1 Patient baseline and disease characteristics

\begin{tabular}{ll}
\hline Characteristic & $N=30$ \\
\hline Median age, years (range) & $61(30-80)$ \\
Sex, $n(\%)$ & $28(93.3)$ \\
Male & $2(6.7)$ \\
Female & \\
ECOG performance status, $n(\%)$ & $5(16.7)$ \\
0 & $25(83.3)$ \\
1 & \\
Number of prior anticancer therapy regimens, $n(\%)$ & $6(20.0)$ \\
1 & $14(46.7)$ \\
2 & $10(33.3)$ \\
$\geq 3$ & \\
Number of prior lines of therapy for locally advanced or & \\
metastatic disease, $n(\%)$ & $5(16.7)$ \\
0 & $14(46.7)$ \\
1 & $11(36.7)$ \\
$\geq 2$ & \\
Type of prior anticancer therapy for locally advanced or & \\
metastatic disease, $n(\%)^{\mathrm{a}}$ & \\
Cytotoxic therapy & $25(83.3)$ \\
Monoclonal antibodies therapy & $6(20.0)$ \\
Small molecules & $2(6.7)$ \\
Tumor cell PD-L1 expression, $n(\%)^{\mathrm{b}}$ & \\
Positive & $9(30.0)$ \\
Negative & $20(66.7)$ \\
Not evaluable & $1(3.3)$ \\
\hline &
\end{tabular}

ECOG Eastern Cooperative Oncology Group

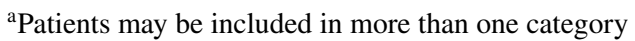

${ }^{\mathrm{b}} \mathrm{A}$ threshold of $1 \%$ was used to characterize tumors as either PD-L1 positive $(\geq 1 \%)$ or negative $(<1 \%)$ using an anti-PD-L1 antibody clone $73-10$

17.3-52.8]). The investigator-assessed confirmed ORR was 13.3\% (95\% CI 3.8-30.7); agreement between independent review committee and investigator-assessed responses is presented in the Online Supplementary Material (OSM), Table S1.

Median PFS was 1.3 months (95\% CI 1.0-5.3) by independent review committee, with a 6 -month rate of $25.9 \%$ (95\% CI 11.5-43.1) (Fig. 2a). Similar median PFS was observed by investigator assessment (1.3 months [95\% CI 1.1-5.3]) (OSM Fig. S1). Median OS was 3.4 months (95\% CI 2.5-10.3), with respective 6- and 12-month OS rates of $39.7 \%$ (95\% CI 22.0-57.0) and 32.1\% (95\% CI 16.0-49.5) (Fig. 2b).

\subsection{Biomarker Analyses}

Response to bintrafusp alfa was observed in patients with PD-L1-positive and PD-L1-negative tumors (Table 2). PD-L1 expression in the TME was also evaluated, and 
Table 2 Treatment response to bintrafusp alfa

\begin{tabular}{lll}
\hline Efficacy $(N=30)$ & Independent review committee & Investigator \\
\hline Confirmed best overall response, $n(\%)$ & & 0 \\
Complete response & 6 & $4(13.3)$ \\
Partial response & $6(20.0)$ & $7(23.3)$ \\
Stable disease & $4(13.3)$ & $12(40.0)$ \\
Progressive disease & $15(50.0)$ & $7(23.3)$ \\
Not evaluable & $5(16.7)$ & $4(13.3)$ \\
Confirmed ORR, $n(\%)$ & $6(20.0)$ & $3.8-30.7$ \\
$95 \%$ CI & $7.7-38.6$ & $11(36.7)$ \\
DCR, $n(\%)$ & $10(33.3)$ & $19.9-56.1$ \\
$95 \%$ CI & $17.3-52.8$ & $\mathrm{NR}(3.6-12.6)$ \\
Median duration of response, months (range) & $4.3(1.3-8.3)$ & $0 / 9(0)$ \\
ORR by tumor cell PD-L1 expression, $n / N(\%)^{\mathrm{a}}$ & & $4 / 20(20.0)$ \\
Positive & $1 / 9(11.1)$ & $0 / 1(0)$ \\
Negative & $4 / 20(20.0)$ & $1 / 1(100)$ \\
Not evaluable & & \\
\hline
\end{tabular}

$D C R$ disease control rate, $N R$ not reached, $O R R$ objective response rate

${ }^{a} \mathrm{~A}$ threshold of $1 \%$ was used to characterize tumors as either PD-L1 positive $(\geq 1 \%)$ or negative $(<1 \%)$ using an anti-PD-L1 antibody clone $73-10$

responses to bintrafusp alfa, as determined by either independent review committee or investigator assessment, were observed independently of PD-L1 expression (data not shown). The majority of responses ( $n=5$ [83.3\%]) occurred in patients with an immune-excluded phenotype (Fig. 3a). The ORR by independent review committee was $12.5 \%$ in the inflamed, $27.8 \%$ in the immune-excluded, and $0 \%$ in the immune-desert phenotype. However, responses to bintrafusp alfa were observed independently of tumor mutation count, as well as expression of genes associated with an active immune pathway (such as $C D 8 A, C D 8 B$, and $I F N G$ ) or TGF- $\beta$ activation (such as TGFB1, TWIST1, VIM) (Fig. 1a, Fig. 3b-g). Further investigation revealed different average levels of tumor TGFBI expression between patients with esophageal $\mathrm{AC}$ in this study and patients with esophageal SCC in a separate phase 1 study of bintrafusp alfa [39], with $41.8 \%$ lower expression in AC than in SCC (false discovery rate-adjusted $p<0.002$ ). TGFB3 expression was also lower in patients with esophageal AC than in those with esophageal SCC, but the difference was not significant (false discovery rate-adjusted $p=0.426$ ). In contrast, TGFB2 expression was $96.6 \%$ higher in esophageal AC than in esophageal SCC (false discovery rate-adjusted $p=0.049$ ) (OSM Fig. S2). When compared across patients from phase 1 trials of bintrafusp alfa, $T G F B$ expression levels in tumor samples from patients with esophageal AC were not significantly different from those from patients with other tumor types with various histologies (false discovery rate-adjusted $p=0.465$ [TGFB1]; $p=0.989$ [TGFB2]; $p=0.082$ [TGFB3]).

\subsection{Safety}

Among the 30 patients, 19 (63.3\%) experienced a TRAE of any grade, of which diarrhea, pruritus ( $n=4$ [13.3\%] each), and asthenia $(n=3[10.0 \%])$ were most common (Table 3$)$. Seven patients (23.3\%) experienced at least one grade 3 TRAE, including anemia $(n=2[6.7 \%])$, Bowen's disease (also known as SCC in situ), cancer pain, gastritis hemorrhagic, hypophysitis, hypopituitarism, rash generalized, and SCC of skin ( $n=1$ [3.3\%] each). No grade 4 TRAEs or treatment-related deaths occurred. TRAEs led to permanent discontinuation in two patients (6.7\%). One patient developed a cough 2 days after the first dose of bintrafusp alfa. The cough persisted, and 4 weeks later the patient developed dyspnea (grade 1), determined as treatment related by the investigator. Imaging showed lymphangitis carcinomatosis and findings that were suggestive of multiple small lung metastases. Bintrafusp alfa was permanently discontinued, and the patient withdrew from the study due to confirmed disease progression. The second patient permanently discontinued treatment due to grade 3 gastritis hemorrhagic, also determined as treatment related by the investigator. This patient had a history of pseudoaneurysm of the celiac trunk and developed treatment-emergent gastritis hemorrhagic 3 weeks after the first study dose of bintrafusp alfa ( 9 days after the preceding dose). Gastrointestinal bleeding of unknown origin was found upon hospitalization. This event improved after 3 weeks following blood transfusions and treatment with parenteral tranexamic acid, somatostatin, and proton pump inhibitors. Following resolution of the AE and 

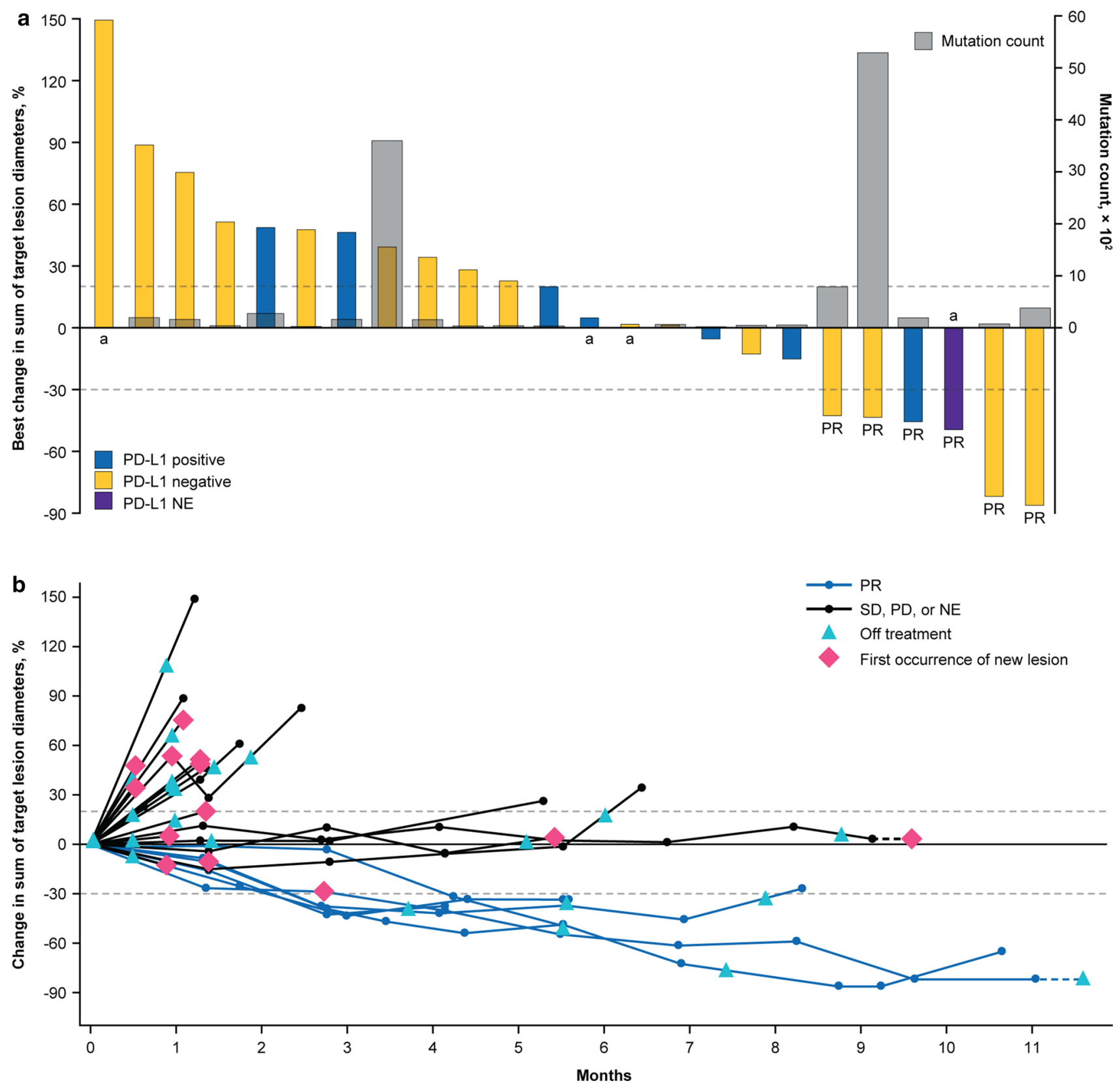

Fig. 1 Tumor response to bintrafusp alfa assessed by independent review. a Best change in sum of diameters and tumor mutation count. A threshold of $1 \%$ was used to characterize tumors as either PD-L1 positive $(\geq 1 \%)$ or negative $(<1 \%)$ using an anti-PD-L1 antibody clone 73-10. One patient had non-evaluable PD-L1 expression. b Time to and duration of response. The upper dashed line represents progression at $20 \%$ increase in size of target lesions, and the lower dashed line represents the RECIST boundary for partial response at 30\% decrease in size of target lesions. Six patients are not shown because they had no post-baseline assessments due to death within 6 weeks after starting treatment $(n=2)$, had no baseline and postbaseline target lesion measurement $(n=1)$, or due to other reasons $(n$ $=3$ ). ${ }^{\text {a }}$ Tumor mutation count data unavailable. $N E$ not evaluable, $P D$ progressive disease, $P R$ partial response, $S D$ stable disease reinitiation of bintrafusp alfa, grade 3 gastritis hemorrhagic recurred and was determined to be related to bintrafusp alfa. The patient permanently discontinued study treatment and received the same medical management that was previously used to treat their gastritis hemorrhagic for 3 weeks until the event resolved. Seven patients $(23.3 \%)$ had irAEs, none of which were above grade 3 or led to discontinuation of treatment (OSM Table S2). No patient experienced a grade $\geq 3$ infusion-related reaction that was assessed by the investigator as being related to bintrafusp alfa. Potentially TGF$\beta$-related skin lesions were reported in two patients $(6.7 \%)$, one of whom experienced multiple events (OSM Table S2). 

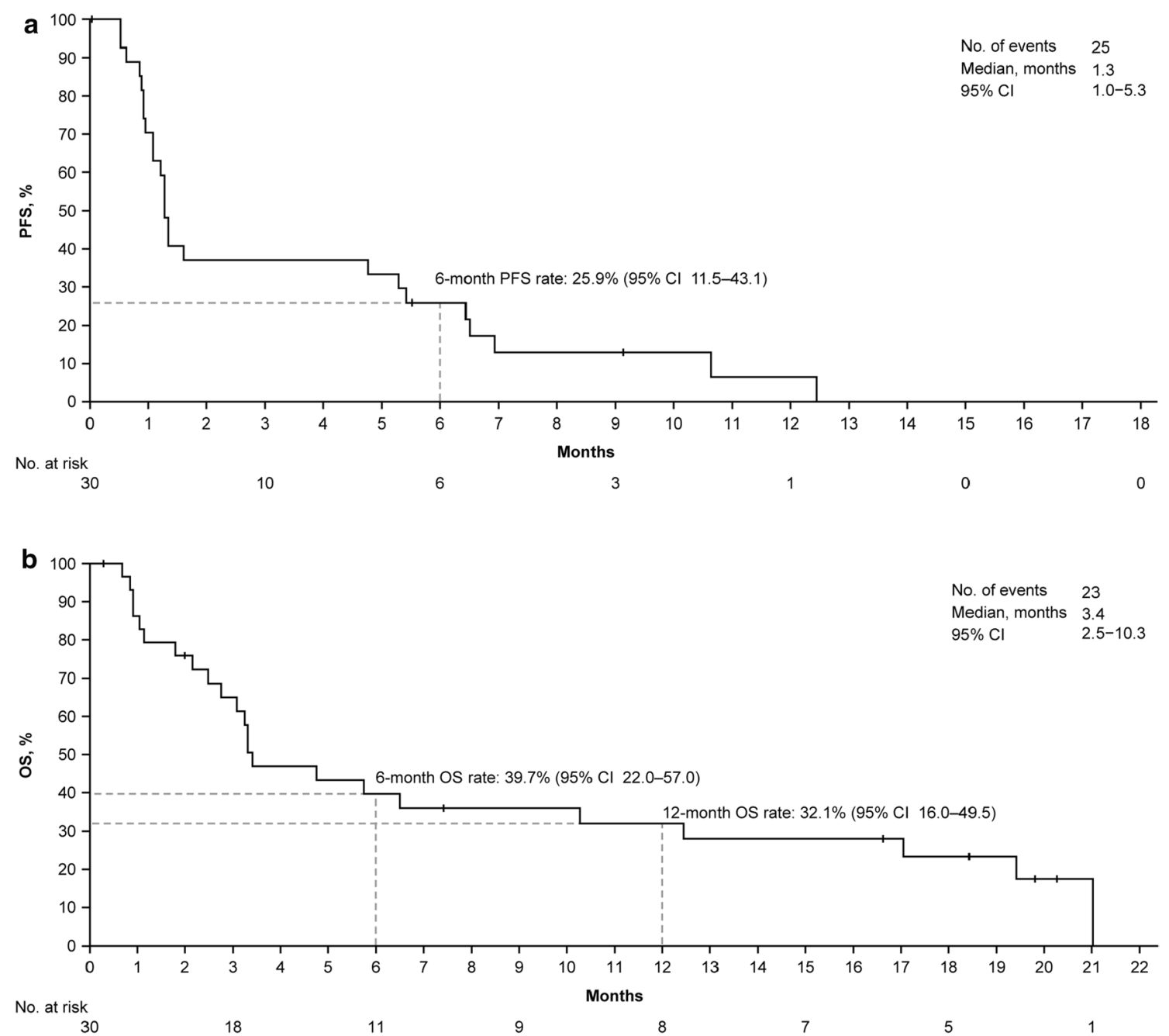

Fig. 2 Survival outcomes. Kaplan-Meier survival curves for a independent review committee-assessed progression-free survival (PFS) and b overall survival (OS)

These skin lesions were generally managed well with complete excision when needed and did not lead to treatment discontinuation.

\section{Discussion}

In this phase 1 , dose-expansion cohort of 30 patients with advanced, post-platinum esophageal AC, bintrafusp alfa showed evidence of clinical activity with an ORR of $20.0 \%$ per independent review committee assessment and a 12 -month OS rate of $32.1 \%$ in the overall, PD-L1-unselected cohort. Additionally, responses were independent of PD-L1 expression on tumor cells or on cells of the TME. Phase 2 and 3 studies of pembrolizumab showed ORRs that ranged from 3 to $18 \%$ and 12-month OS rates that were between 22 and $24 \%$ in patients with pretreated esophageal AC, depending on PD-L1 expression [14, 15]. However, the results observed here for bintrafusp alfa cannot be directly compared with those reported for pembrolizumab because of key differences in study design. Larger studies are needed to confirm the impact of PD-L1 expression on clinical activity with bintrafusp alfa in esophageal AC.

Results of exploratory analyses showed that response to bintrafusp alfa was independent of tumor mutation count. Patients in this esophageal AC cohort had lower mutation counts than patients with esophageal SCC enrolled in a different phase 1 study of bintrafusp alfa (NCT02699515) [39]. Furthermore, the average expression levels of $T G F B 1$, $T G F B 2$, and $T G F B 3$ in tumor samples from patients in this study were similar to those from patients with other tumors across phase 1 studies of bintrafusp alfa; however, the levels of $T G F B 1$ and $T G F B 2$ were significantly different (lower and higher, respectively) than those observed in an esophageal SCC cohort of the separate phase 1 study of bintrafusp alfa. Previous reports have shown a link between TGF- $\beta$ 

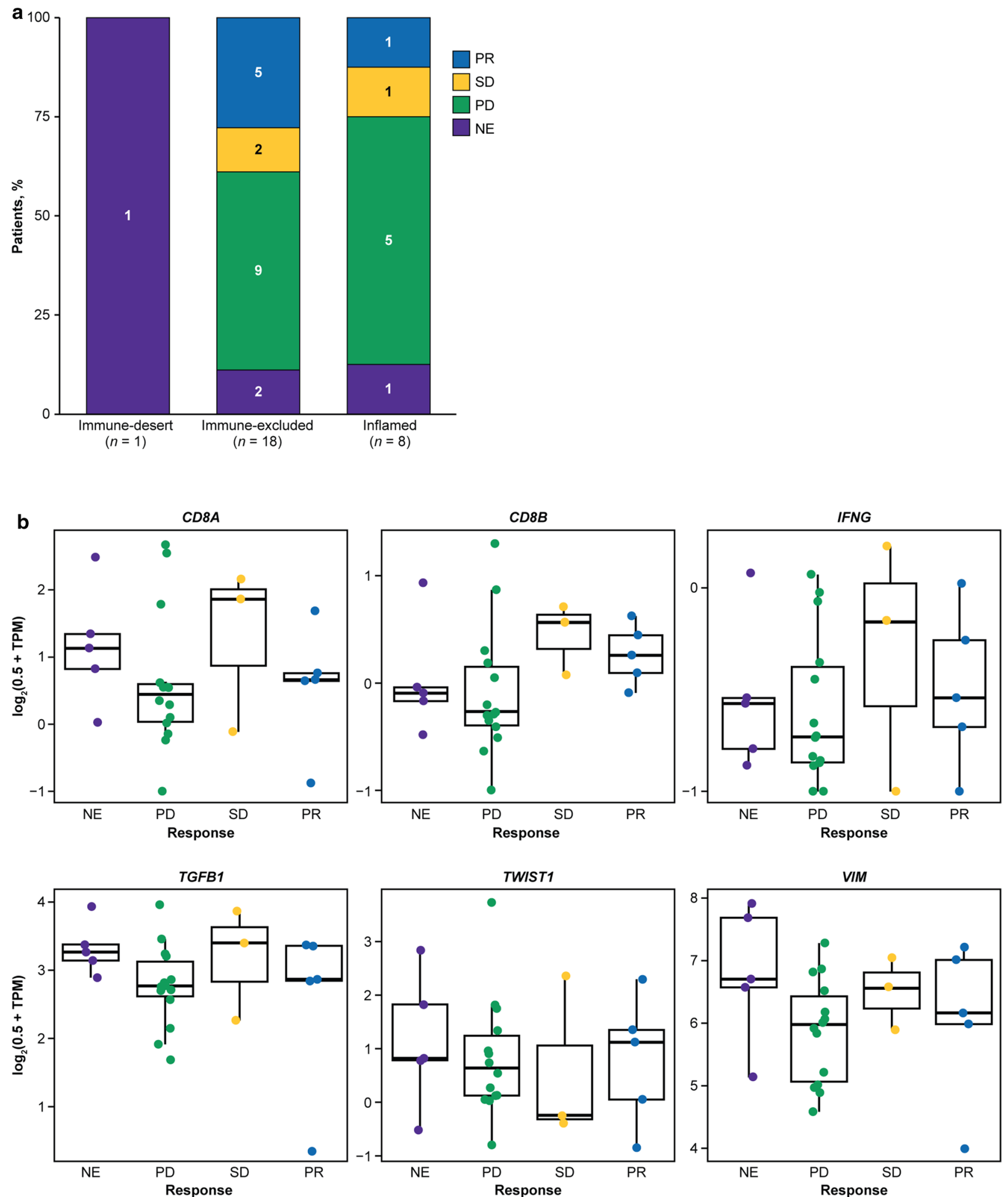

Fig. 3 Exploratory biomarker analysis by confirmed best overall response to bintrafusp alfa per independent review committee assessment. a Immune phenotype analysis; three patients with immune phenotypes of indeterminate $(n=1)$ or not annotated $(n=2)$ are not shown. b Gene expression analysis; three patients with confirmed best overall responses of partial response, stable disease, and progres- sive disease ( $n=1$, each) were not included in the RNAseq analysis due to failing QC or lack of RNAseq data. $I F N G$ interferon gamma, $N E$ not evaluable, $P D$ progressive disease, $P R$ partial response, $S D$ stable disease, TGFB1 transforming growth factor beta 1, TPM transcript per million, TWIST1 twist family bHLH transcription factor 1, VIM vimentin 
Table 3 TRAEs occurring at any grade in $\geq 5 \%$ of patients or at grade 3 severity, and any AEs of special interest

\begin{tabular}{lll}
\hline Preferred term, $n(\%)$ & $N=30$ & \\
\cline { 2 - 3 } & Any grade & Grade $^{\mathrm{a}}$ \\
\hline TRAEs & & \\
TRAE & $19(63.3)$ & $7(23.3)$ \\
Diarrhea & $4(13.3)$ & 0 \\
Pruritus & $4(13.3)$ & 0 \\
Asthenia & $3(10.0)$ & 0 \\
Anemia & $2(6.7)$ & $2(6.7)$ \\
Rash generalized & $2(6.7)$ & $1(3.3)$ \\
Alanine aminotransferase increased & $2(6.7)$ & 0 \\
Aspartate aminotransferase increased & $2(6.7)$ & 0 \\
Dyspnea & $2(6.7)$ & 0 \\
Fatigue & $2(6.7)$ & 0 \\
Hyperthyroidism & $2(6.7)$ & 0 \\
KA & $2(6.7)$ & 0 \\
Nausea & $2(6.7)$ & 0 \\
Rash & $2(6.7)$ & 0 \\
Rash, macular & $2(6.7)$ & 0 \\
Bowen's disease ${ }^{\text {b }}$ & $1(3.3)$ & $1(3.3)$ \\
Cancer pain & $1(3.3)$ & $1(3.3)$ \\
Gastritis hemorrhagic & $1(3.3)$ & $1(3.3)$ \\
Hypophysitis & $1(3.3)$ & $1(3.3)$ \\
Hypopituitarism & $1(3.3)$ & $1(3.3)$ \\
SCC of skin & $1(3.3)$ & $1(3.3)$ \\
Any AE of special interest & $2(6.7)$ & $1(3.3)$ \\
Skin lesions & & \\
Any irAE & $7(23.3)^{\mathrm{d}}$ & $2(6.7)$ \\
Immune-related rash & $5(16.7)$ & $1(3.3)$ \\
Immune-related pituitary dysfunction & $1(3.3)$ & $1(3.3)$ \\
Immune-related pneumonitis & $1(3.3)$ & 0 \\
Immune-related thyroid disorders & $1(3.3)$ & 0 \\
\hline
\end{tabular}

$A E$ adverse event, $i r A E$ immune-related adverse event, $K A$ keratoacanthoma, SCC squamous cell carcinoma, TRAE treatment-related adverse event

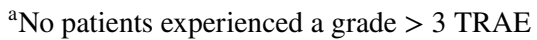

${ }^{\mathrm{b}}$ Also known as SCC in situ

${ }^{\mathrm{c}}$ Includes actinic keratosis, basal cell carcinoma, Bowen's disease, hyperkeratosis, KA, lip SCC, and SCC of skin NCI-CTCAE v4.03 preferred terms

${ }^{\mathrm{d}}$ Three patients experienced multiple different irAEs

and immune phenotype, suggesting TGF- $\beta$ can restrict T-cell infiltration in the TME and limit antitumor immunity [16, 40]. In preclinical studies, the combined use of a TGF- $\beta$ inhibitor with a PD-L1 inhibitor significantly increased tumor-infiltrating $\mathrm{T}$ cells and significantly reduced tumor burden in mouse models of immune-excluded tumors [16]. Interestingly, all but one response occurred in immuneexcluded tumors in this esophageal AC cohort. Similar results were also observed in patients with esophageal SCC from the other study, in which responses were seen exclusively in immune-excluded tumors [39]. Taken together, the results of the exploratory analyses did not identify predictive biomarkers of response to bintrafusp alfa in this small cohort.

Treatment with bintrafusp alfa was well tolerated in this patient population, with no grade 4 TRAEs or treatmentrelated deaths. The safety profile, including incidence of irAEs, was similar to that seen with checkpoint inhibitors, except for potentially TGF- $\beta$-related skin lesions, which were reported in two patients [14, 15, 41-48]. These AEs were generally managed well and did not lead to discontinuation from the trial. Importantly, skin lesions, such as keratoacanthomas, are anticipated AEs for TGF- $\beta$ inhibition [49]. The overall safety profile reported here for bintrafusp alfa in esophageal AC was consistent with those previously observed with bintrafusp alfa treatment in other solid tumors, including esophageal SCC [23, 24, 39].

Limitations of this study include the small enrollment size and the lack of a comparator arm, which preclude any definitive conclusions regarding comparisons of bintrafusp alfa with other available treatment options.

The clinical activity and manageable safety profile observed in this study of heavily pretreated patients with esophageal AC suggest that bintrafusp alfa could provide a novel treatment approach for these patients. Further clinical investigation of bintrafusp alfa in esophageal cancer is warranted based upon these results.

Supplementary Information The online version contains supplementary material available at https://doi.org/10.1007/s11523-021-00809-2.

Acknowledgements The authors thank the patients and their families, investigators, co-investigators, and study teams at each of the participating centers and at Merck KGaA, Darmstadt, Germany, and EMD Serono Research \& Development Institute, Inc., Billerica, MA, USA; an affiliate of Merck KGaA, Darmstadt, Germany. The authors thank Christian Ihling, of Merck KGaA, and George Locke, of EMD Serono Research \& Development Institute, Inc., for their substantial contribution to the immune phenotype and biomarker analysis, respectively. Medical writing support was provided by Lauren Rusnak, $\mathrm{PhD}$, of ClinicalThinking Inc, Hamilton, NJ, USA, which was also funded by Merck KGaA and GlaxoSmithKline in accordance with Good Publication Practice (GPP3) guidelines (http://www.ismpp.org/gpp3).

Data availability For all new products or new indications approved in both the European Union and the USA after 1 January 2014, Merck KGaA, Darmstadt, Germany, will share patient-level and study-level data after deidentification, as well as redacted study protocols and clinical study reports from clinical trials in patients. Any requests for these data by qualified scientific and medical researchers for legitimate research purposes will be subject to Merck KGaA's Data Sharing Policy. All requests should be submitted in writing to Merck KGaA's data sharing portal (https://www.merckgroup.com/en/research/our-appro ach-to-research-and-development/healthcare/clinical-trials/commi tment-responsible-data-sharing.html). When Merck KGaA has a coresearch, co-development, or co-marketing or co-promotion agreement, 
or when the product has been out-licensed, the responsibility for disclosure might be dependent on the agreement between parties. Under these circumstances, Merck KGaA will endeavor to gain agreement to share data in response to requests.

\section{Declarations}

Funding This trial was funded by Merck KGaA, Darmstadt, Germany, and is part of an alliance between Merck KGaA and GlaxoSmithKline. Merck KGaA provided the study drug and worked with investigators on the trial design and plan, collection and analysis of data, and interpretation of results. Funding for a professional medical writer with access to the data was provided by Merck KGaA and GlaxoSmithKline.

Conflicts of interest/Competing interest B. Tan received research grants from EMD Serono Research \& Development Institute, Inc., Billerica, MA, USA; an affiliate of Merck KGaA, Darmstadt, Germany. A. Khattak has consulted for Merck Healthcare Pty. Ltd., Australia; an affiliate of Merck KGaA, Darmstadt, Germany; received research grants from Merck Sharp \& Dohme; and reports honoraria from Bayer, Bristol Myers Squibb, Merck Sharp \& Dohme, Pfizer, Merck Healthcare Pty. Ltd., and Novartis. E. Felip has consulted for AbbVie, AstraZeneca, Blueprint Medicines, Boehringer Ingelheim, Bristol Myers Squibb, Eli Lilly, Guardant Health, Janssen, Merck KGaA, Darmstadt, Germany, Merck Sharp \& Dohme, Novartis, Pfizer, Roche, Samsung, Takeda, and GlaxoSmithKline; has received research grants from Fundación Merck Salud; reports honoraria from AstraZeneca, Boehringer Ingelheim, Bristol Myers Squibb, Eli Lilly, prIME Oncology, Medscape, and touchIME; and is an independent member of the Grifols board. K. Kelly has consulted for AbbVie, AstraZeneca, Bristol Myers Squibb, EMD Serono Research \& Development Institute, Inc., Billerica, MA, USA; an affiliate of Merck KGaA, Darmstadt, Germany, Eli Lilly, Inviata, Novartis, Regeneron, and Takeda; received research grants and other research support from AbbVie, Bristol Myers Squibb, Celgene, EMD Serono Research \& Development Institute, Inc., Five Prime, Genentech, Eli Lilly, Lycera, Merck, Novartis, Regeneron, Tizona, and Transgene; reports honoraria from Merck; and reports royalties from UpToDate. P. Rich is an employee of Cancer Treatment Centers of America. D. Wang declares no competing interests. C. Helwig is an employee of and has ownership interest in Merck KGaA, Darmstadt, Germany. I. Dussault is an employee of EMD Serono Research \& Development Institute, Inc., Billerica, MA, USA; an affiliate of Merck KGaA, Darmstadt, Germany; and has ownership interest in EMD Serono Research \& Development Institute, Inc., and Amgen. L.S. Ojalvo is an employee of and reports intellectual property interests with EMD Serono Research \& Development Institute, Inc., Billerica, MA, USA; an affiliate of Merck KGaA, Darmstadt, Germany. N. Isambert has consulted for Ipsen and Transgene.

Ethics approval and consent to participate The protocol for this study was approved by all relevant regulatory authorities and ethics committees at participating institutions, and the study was conducted in accordance with the International Conference on Harmonisation Topic E6 Good Clinical Practice and the Declaration of Helsinki. Each patient provided written informed consent before study enrollment.

Consent for publication All authors gave final approval of the version to be published.

Code Availability Not applicable.

Author contributions $\mathrm{CH}$, ID, and LSO contributed to study conception and design; BT, AK, EF, KK, PR, DW, and NI contributed to data acquisition. All authors contributed to data interpretation and drafting of the manuscript. All authors reviewed and approved the final manuscript and approved the final manuscript for publication.

Open Access This article is licensed under a Creative Commons Attribution-NonCommercial 4.0 International License, which permits any non-commercial use, sharing, adaptation, distribution and reproduction in any medium or format, as long as you give appropriate credit to the original author(s) and the source, provide a link to the Creative Commons licence, and indicate if changes were made. The images or other third party material in this article are included in the article's Creative Commons licence, unless indicated otherwise in a credit line to the material. If material is not included in the article's Creative Commons licence and your intended use is not permitted by statutory regulation or exceeds the permitted use, you will need to obtain permission directly from the copyright holder. To view a copy of this licence, visit $\mathrm{http} / / /$ creativecommons.org/licenses/by-nc/4.0/.

\section{References}

1. Bray F, Ferlay J, Soerjomataram I, Siegel RL, Torre LA, Jemal A. Global cancer statistics 2018: GLOBOCAN estimates of incidence and mortality worldwide for 36 cancers in 185 countries. CA Cancer J Clin. 2018;68(6):394-424.

2. Allemani C, Matsuda T, Di Carlo V, Harewood R, Matz M, Nikšić $\mathrm{M}$, et al. Global surveillance of trends in cancer survival 2000-14 (CONCORD-3): analysis of individual records for 37513025 patients diagnosed with one of 18 cancers from 322 populationbased registries in 71 countries. Lancet. 2018;391(10125):102375. https://doi.org/10.1016/S0140-6736(17)33326-3.

3. Zhang Y. Epidemiology of esophageal cancer. World J Gastroenterol. 2013;19(34):5598-606.

4. National Comprehensive Cancer Network. NCCN Clinical Practice Guidelines in Oncology (NCCN guidelines): Esophageal and Esophagogastric Junction Cancers. V4.2020. 2020.

5. The Cancer Genome Atlas Research Network, Kim J, Bowlby R, Mungall AJ, Robertson AG, Odze RD, et al. Integrated genomic characterization of oesophageal carcinoma. Nature. 2017;541:169-75.

6. Lin EW, Karakasheva TA, Hicks PD, Bass AJ, Rustgi AK. The tumor microenvironment in esophageal cancer. Oncogene. 2016;35:5337.

7. Achyut BR, Yang L. Transforming growth factor- $\beta$ in the gastrointestinal and hepatic tumor microenvironment. Gastroenterology. 2011;141(4):1167-78. https://doi.org/10.1053/j.gastro.2011.07. 048.

8. Principe DR, Doll JA, Bauer J, Jung B, Munshi HG, Bartholin L, et al. TGF- $\beta$ : duality of function between tumor prevention and carcinogenesis. J Natl Cancer Inst. 2014;106(2):djt369.

9. Akhurst RJ, Hata A. Targeting the TGF $\beta$ signalling pathway in disease. Nat Rev Drug Discov. 2012;11(10):790-811.

10. Colak S, ten Dijke P. Targeting TGF- $\beta$ signaling in cancer. Trends Cancer. 2017;3(1):56-71. https://doi.org/10.1016/j.trecan.2016. 11.008 .

11. Rees JRE, Onwuegbusi BA, Save VE, Alderson D, Fitzgerald $\mathrm{RC}$. In vivo and in vitro evidence for transforming growth factor$\beta 1$-mediated epithelial to mesenchymal transition in esophageal adenocarcinoma. Cancer Res. 2006;66(19):9583-90.

12. Ebbing EA, Steins A, Fessler E, Stathi P, Lesterhuis WJ, Krishnadath KK, et al. Esophageal adenocarcinoma cells and xenograft tumors exposed to Erb-b2 receptor tyrosine kinase 2 and 3 inhibitors activate transforming growth factor beta signaling, which induces epithelial to mesenchymal transition. Gastroenterology. 2017;153(1):63-76.e14. 
13. von Rahden BHA, Stein HJ, Feith M, Pühringer F, Theisen J, Siewert JR, et al. Overexpression of TGF- $\beta 1$ in esophageal (Barrett's) adenocarcinoma is associated with advanced stage of disease and poor prognosis. Mol Carcinog. 2006;45(10):786-94.

14. Shah MA, Kojima T, Hochhauser D, Enzinger P, Raimbourg J, Hollebecque A, et al. Efficacy and safety of pembrolizumab for heavily pretreated patients with advanced, metastatic adenocarcinoma or squamous cell carcinoma of the esophagus: the phase 2 KEYNOTE-180 study. JAMA Oncol. 2019;5(4):546-50.

15. Kojima T, Shah MA, Muro K, Francois E, Adenis A, Hsu C-H, et al. Randomized phase III KEYNOTE-181 study of pembrolizumab versus chemotherapy in advanced esophageal cancer. J Clin Oncol. 2020;38(35):4138-48.

16. Mariathasan S, Turley SJ, Nickles D, Castiglioni A, Yuen K, Wang $\mathrm{Y}$, et al. TGF $\beta$ attenuates tumour response to PD-L1 blockade by contributing to exclusion of T cells. Nature. 2018;554:544-8.

17. Holmgaard RB, Schaer DA, Li Y, Castaneda SP, Murphy MY, $\mathrm{Xu} \mathrm{X}$, et al. Targeting the TGF $\beta$ pathway with galunisertib, a TGF $\beta R I$ small molecule inhibitor, promotes anti-tumor immunity leading to durable, complete responses, as monotherapy and in combination with checkpoint blockade. J ImmunoTher Cancer. 2018;6(1):47.

18. Tauriello DVF, Palomo-Ponce S, Stork D, Berenguer-Llergo A, Badia-Ramentol J, Iglesias M, et al. TGF $\beta$ drives immune evasion in genetically reconstituted colon cancer metastasis. Nature. 2018;554:538-43.

19. Principe DR, Park A, Dorman MJ, Kumar S, Viswakarma N, Rubin J, et al. TGF $\beta$ blockade augments PD-1 inhibition to promote T-cell-mediated regression of pancreatic cancer. Mol Cancer Ther. 2019;18(3):613-20.

20. Lan Y, Zhang D, Xu C, Hance KW, Marelli B, Qi J, et al. Enhanced preclinical antitumor activity of M7824, a bifunctional fusion protein simultaneously targeting PD-L1 and TGF- $\beta$. Sci Transl Med. 2018;10(424):eaan5488.

21. Knudson KM, Hicks KC, Luo X, Chen JQ, Schlom J, Gameiro SR. M7824, a novel bifunctional anti-PD-L1/TGF $\beta$ Trap fusion protein, promotes anti-tumor efficacy as monotherapy and in combination with vaccine. Oncoimmunology. 2018;7(5):e1426519.

22. David JM, Dominguez C, McCampbell KK, Gulley JL, Schlom J, Palena C. A novel bifunctional anti-PD-L1/TGF- $\beta$ Trap fusion protein (M7824) efficiently reverts mesenchymalization of human lung cancer cells. Oncoimmunology. 2017;6(10):e1349589-e.

23. Strauss J, Heery CR, Schlom J, Madan RA, Cao L, Kang Z, et al. Phase I trial of M7824 (MSB0011359C), a bifunctional fusion protein targeting PD-L1 and TGF $\beta$, in advanced solid tumors. Clin Cancer Res. 2018;24(6):1287-95.

24. Doi T, Fujiwara Y, Koyama T, Ikeda M, Helwig C, Watanabe M, et al. Phase I study of the bifunctional fusion protein bintrafusp alfa in Asian patients with advanced solid tumors, including a hepatocellular carcinoma safety-assessment cohort. Oncologist. 2020;25(9):e1292-302.

25. Tan B, Khattak A, Felip E, Kelly K, Wang D, Helwig C, et al. M7824 (MSB0011359C), a bifunctional fusion protein targeting PD-L1 and TGF- $\beta$, in patients with post-platinum esophageal adenocarcinoma (EAC): preliminary results from a phase 1 cohort. Ann Oncol. 2018;29(Suppl 8):Abstract-643P.

26. Vugmeyster Y, Wilkins J, Koenig A, El Bawab S, Dussault I, Ojalvo LS, et al. Selection of the recommended phase 2 dose for bintrafusp alfa, a bifunctional fusion protein targeting TGF- $\beta$ and PD-L1. Clin Pharmacol Ther. 2020;108(3):566-74.

27. Wilkins JJ, Vugmeyster Y, Dussault I, Girard P, Khandelwal A. Population pharmacokinetic analysis of bintrafusp alfa in different cancer types. Adv Ther. 2019;36(9):2414-33. https://doi.org/10. 1007/s12325-019-01018-0.

28. Langmead B, Salzberg SL. Fast gapped-read alignment with Bowtie 2. Nat Methods. 2012;9(4):357-9.
29. Li B, Dewey CN. RSEM: accurate transcript quantification from RNA-Seq data with or without a reference genome. BMC Bioinformatics. 2011;12(1):323.

30. Ritchie ME, Phipson B, Wu D, Hu Y, Law CW, Shi W, et al. limma powers differential expression analyses for RNA-sequencing and microarray studies. Nucleic Acids Res. 2015;43(7):e47.

31. Dobin A, Davis CA, Schlesinger F, Drenkow J, Zaleski C, Jha S, et al. STAR: ultrafast universal RNA-seq aligner. Bioinformatics. 2012;29(1):15-21.

32. Li H, Durbin R. Fast and accurate short read alignment with Burrows-Wheeler transform. Bioinformatics. 2009;25(14):1754-60.

33. Lai Z, Markovets A, Ahdesmaki M, Chapman B, Hofmann O, McEwen R, et al. VarDict: a novel and versatile variant caller for next-generation sequencing in cancer research. Nucleic Acids Res. 2016;44(11):e108. https://doi.org/10.1093/nar/gkw227.

34. McLaren W, Gil L, Hunt SE, Riat HS, Ritchie GR, Thormann A, et al. The Ensembl Variant Effect Predictor. Genome Biol. 2016;17(1):122.

35. Chen DS, Mellman I. Elements of cancer immunity and the cancer-immune set point. Nature. 2017;541:321-30.

36. Kim JM, Chen DS. Immune escape to PD-L1/PD-1 blockade: seven steps to success (or failure). Ann Oncol. 2016;27(8):1492-504.

37. Hegde PS, Karanikas V, Evers S. The where, the when, and the how of immune monitoring for cancer immunotherapies in the era of checkpoint inhibition. Clin Cancer Res. 2016;22(8):1865-74.

38. Bacac M, Fauti T, Sam J, Colombetti S, Weinzierl T, Ouaret D, et al. A novel carcinoembryonic antigen T-cell bispecific antibody (CEA TCB) for the treatment of solid tumors. Clin Cancer Res. 2016;22(13):3286-97. https://doi.org/10.1158/1078-0432. ccr-15-1696.

39. Lin C-C, Doi T, Muro K, Hou M-M, Esaki T, Hara H, et al. Bintrafusp alfa, a bifunctional fusion protein targeting TGF- $\beta$ and PD-L1, in patients with esophageal squamous cell carcinoma: results from a phase 1 cohort in Asia. Target Oncol. 2021. https:// doi.org/10.1007/s11523-021-00810-9

40. Hegde PS, Chen DS. Top 10 challenges in cancer immunotherapy. Immunity. 2020;52(1):17-35.

41. Ralph C, Elkord E, Burt DJ, O'Dwyer JF, Austin EB, Stern PL, et al. Modulation of lymphocyte regulation for cancer therapy: A phase II trial of tremelimumab in advanced gastric and esophageal adenocarcinoma. Clin Cancer Res. 2010;16(5):1662-72.

42. Doi T, Piha-Paul SA, Jalal SI, Saraf S, Lunceford J, Koshiji M, et al. Safety and antitumor activity of the anti-programmed death-1 antibody pembrolizumab in patients with advanced esophageal carcinoma. J Clin Oncol. 2018;36(1):61-7.

43. Shen J, Chang J, Mendenhall M, Cherry G, Goldman JW, Kulkarni RP. Diverse cutaneous adverse eruptions caused by anti-programmed cell death-1 (PD-1) and anti-programmed cell death ligand-1 (PD-L1) immunotherapies: clinical features and management. Ther Adv Med Oncol. 2018;10:1758834017751634.

44. Bang YJ, Ruiz EY, Van Cutsem E, Lee KW, Wyrwicz L, Schenker M, et al. Phase III, randomised trial of avelumab versus physician's choice of chemotherapy as third-line treatment of patients with advanced gastric or gastro-oesophageal junction cancer: primary analysis of JAVELIN Gastric 300. Ann Oncol. 2018;29(10):2052-60.

45. Khoja L, Day D, Wei-Wu Chen T, Siu LL, Hansen AR. Tumourand class-specific patterns of immune-related adverse events of immune checkpoint inhibitors: a systematic review. Ann Oncol. 2017;28(10):2377-85.

46. Fuchs CS, Doi T, Jang RW, Muro K, Satoh T, Machado M, et al. Safety and efficacy of pembrolizumab monotherapy in patients with previously treated advanced gastric and gastroesophageal junction cancer: phase 2 clinical KEYNOTE-059 trial. JAMA Oncol. 2018;4:e180013. 
47. Shitara K, Özgüroğlu M, Bang Y-J, Di Bartolomeo M, Mandalà M, Ryu M-H, et al. Pembrolizumab versus paclitaxel for previously treated, advanced gastric or gastro-oesophageal junction cancer (KEYNOTE-061): a randomised, open-label, controlled, phase 3 trial. Lancet. 2018;392(10142):123-33.

48. Kang Y-K, Boku N, Satoh T, Ryu M-H, Chao Y, Kato K, et al. Nivolumab in patients with advanced gastric or gastro-oesophageal junction cancer refractory to, or intolerant of, at least two previous chemotherapy regimens (ONO-4538-12, ATTRAC TION-2): a randomised, double-blind, placebo-controlled, phase 3 trial. Lancet. 2017;390(10111):2461-71.
49. Lacouture ME, Morris JC, Lawrence DP, Tan AR, Olencki TE, Shapiro GI, et al. Cutaneous keratoacanthomas/squamous cell carcinomas associated with neutralization of transforming growth factor $\beta$ by the monoclonal antibody fresolimumab (GC1008). Cancer Immunol Immunother. 2015;64(4):437-46.

\section{Authors and Affiliations}

\section{Benjamin $\operatorname{Tan}^{1} \cdot$ Adnan Khattak ${ }^{2}$ - Enriqueta Felip ${ }^{3} \cdot$ Karen Kelly $^{4}$ Patricia Rich ${ }^{5,6}$. Ding Wang ${ }^{7} \cdot$ Christoph Helwig $^{8}$. Isabelle Dussault ${ }^{9,10} \cdot$ Laureen S. Ojalvo $^{9,10} \cdot$ Nicolas Isambert $^{11}$}

1 Washington University School of Medicine, St Louis, MO, USA

2 Fiona Stanley Hospital, Perth, WA, Australia

3 Vall d'Hebron University Hospital, Vall d'Hebron Institute of Oncology (VHIO), IOB-Quiron, UVic-UCC, Barcelona, Spain

4 University of California Davis Comprehensive Cancer Center, Sacramento, CA, USA

5 Cancer Treatment Centers of America, Atlanta, GA, USA

6 Present Address: Piedmont Healthcare, Atlanta, GA, USA
7 Henry Ford Cancer Institute, Detroit, MI, USA

8 Merck KGaA, Darmstadt, Germany

9 EMD Serono Research \& Development Institute, Inc., Billerica, MA, USA

10 An affiliate of Merck KGaA, Darmstadt, Germany

11 Poitiers University Hospital, 2 rue de la Miléterie, BP 577, 86021 Poitiers, France 